\title{
Heuristische Untersuchung der Abhängigkeit von Übungen und Vorlesung für den Prüfungserfolg
}

\author{
Corinna Modiz ${ }^{* 1}$, Franziska Gorgas ${ }^{1}$, Stefanie Winkler ${ }^{1}$, Andreas Körner ${ }^{1}$ \\ ${ }^{1}$ Inst. of Analysis and Scientific Computing, Vienna University of Technology, Wiedner Haupstraße 8-10, \\ 1040 Vienna, Austria; *corinna.modiz@tuwien.ac.at
}

\begin{abstract}
In dieser Studie werden unterschiedliche Jahrgänge und deren Leistungen abhängig von dem Lehrveranstaltungskonzept untersucht. Dabei wurde eine mathematische Grundlehrveranstaltung bezogen auf den Prüfungserfolg in Abhängigkeit der Leistung in den zugehörigen freiwilligen Übungen evaluiert. Dabei wird gezeigt, dass der Jahrgang mit statischen Aufgaben schlechter abschneidet, als die Jahrgänge mit variierten online Beispielen.
\end{abstract}

\section{Einführung}

Das Erlernen von Fähigkeiten und Fertigkeiten auf einer Universität stützt sich im Grunde auf drei Schwerpunkte. Erstens, das Besuchen der Vorlesung, wobei aktives Zuhören und Mitarbeiten von Vorteil ist. Zweitens, regelmäßiges Anwenden der theoretischen Inhalte in Form von Übungsaufgaben, in denen jene Inhalte auf verschiedene Fragestellungen, beschränkt auf kleinere Teilgebiete angewandt werden [1]. Drittens, das Selbststudium, welches ein Auseinandersetzen mit den Inhalten der Vorlesung und Übung inkludiert, mit dem Ziel den Inhalt gut zu verstehen oder zumindest ein Formulieren jeglicher Unklarheiten ermöglicht [2]. Diese drei Schwerpunkte sind maßgeblich für die Gestaltung der Lehrveranstaltung und sollten daher für den Vortragenden ausschlaggebend sein. Sie sollten bei der Planung und Durchführung des Kurses beachtet werden und Studierenden die Möglichkeit zum Mitlernen und Üben garantieren.

Im folgenden Kapitel wird, basierend auf diesem Grundprinzip, das Konzept der ausgewählten Lehrveranstaltung beschrieben. Dabei werden die unterschiedlichen Umsetzungen der begleitenden freiwilligen Übung erläutert. Anschließend werden die Lehrveranstaltungsergebnisse der Jahrgänge 2016-2019 in Abhängigkeit der drei unterschiedlichen Umsetzungen des Lehrkonzepts unterschieden. Die gesammelten Daten ermöglichen einen direkten Vergleich zwischen dem Einsatz von statischen und variierenden online Beispielen. Im Conclusio werden die Schlussfolgerungen der Datenevaluierung zusammengefasst wiedergegeben.

\section{Lehrkonzept \& Tools}

An der TU Wien wird im Bereich der Grundausbildung in Mathematik für Ingenieurswissenschaften seit einigen Jahren ein Lehrkonzept angewendet, welches Studierenden das Erlernen von mathematischen Fähigkeiten, basierend auf den oben genannten Grundlagen, ermöglicht. Neben einer Vorlesung, die frontal vorgetragen wird, umfassen Lehrveranstaltungen, welche sich der mathematischen Grundausbildung von Ingenieuren widmen, zusätzlich auch eine wöchentlich abgehaltene Präsenzübung. In den meisten Lehrveranstaltungen dieser Art, wird für die Übungsaufgaben das Online-System Möbius (ehemalig Maple T.A.) zur Verfügung gestellt. Diese Plattform basiert auf dem Computer-Algebra-System Maple und ermöglicht, sowohl Variablen sowie auch Funktionen in einem Beispiel einfach zu randomisieren, als auch eine automatisierte Bewertung der Aufgaben. Über die letzten Jahre wurden etliche Beispiele zu verschiedenen praktischen sowie theoretischen Fragestellungen entwickelt. Mit der Randomisierung der Beispiele haben Studierende die Möglichkeit, zu unterschiedlichen Lehrinhalten eine Vielzahl an Beispielen zu üben [3].

Verwendung findet Möbius in den Lehrveranstaltungen sowohl in Form von Übungssammlungen zum freien Üben, als auch zur Leistungsüberprüfung bei Klausuren und Vorlesungsprüfungen. Die Bepunktung der Beispiele wird voreingestellt, wodurch die Benotung für alle Teilnehmer gleich und fair durchgeführt werden kann. Bei den Lehrveranstaltungen handelt es sich um Mathematikvorlesungen für Studierende der Elektrotechnik, Geodäsie und Geoinformation, Raumplanung und Technischen Physik. Für diesen Beitrag, wurde die Vorlesung „Mathematische und statistische Grundlagen der Raumplanung“ ausgewählt, welche im nächsten Abschnitt näher beschrieben wird. 


\section{Lehrveranstaltungsmodus}

Die Vorlesung „Mathematische und statistische Grundlagen der Raumplanung“ wird im zweiten Semester des Bachelorstudiums für Raumplanung angeboten. Ursprünglich wurde diese Vorlesung ohne eine zugehörige Übung angeboten. Nachdem die Arbeitsgruppe „Mathematics in Simulation and Education“ die Vorlesung mit Kollegen aus der Raumplanung übernommen hatte, wurde eine begleitende Übung eingeführt. Diese ist allerdings ein freiwilliges Zusatzangebot. An den anderen Ingenieursdisziplinen werden verpflichtende Übungen zu den Mathematiklehrveranstaltungen angeboten.

Die Vorlesung wurde in den angegebenen Jahren als zweistündige Präsenzveranstaltung mit Tafel bzw. Folien abgehalten. Die zugehörige Übung bestand in jedem der Jahrgänge aus den folgenden Komponenten:

- wöchentliche Übungen,

- Tafelleistungen,

- Klausuren und

- Mitarbeit.

In den nachstehenden Abschnitten werden die Unterschiede der einzelnen Jahrgänge herausgearbeitet.

\subsection{Konzeptbeschreibung 2016}

Die begleitende Übung wurde wöchentlich als Präsenzlehrveranstaltung durchgeführt. Für die Übungsvorbereitung wurden übungsrelevante Beispiele der Möbius Plattform in Moodle als Link zur Verfügung gestellt. In den Präsenzeinheiten wurden diese Beispiele von dem/der Übungsleitung vorgerechnet. Studierende hatten die Möglichkeit, vorbereitete Beispiele selbst vorzuführen bzw. mitzuarbeiten, um Bonuspunkte für die Abschlussnote zu sammeln. Im Laufe des Semesters wurden drei Klausuren angeboten, von denen mindestens zwei positiv abgelegt werden mussten. Zusätzlich gab es auch eine Mindestpunktezahl in jeder Klausur. Ein weiteres Angebot waren die Hausübungen, welche eine Woche vor den Klausuren online gestellt wurden und als Vorbereitung gedacht waren. Die Ergebnisse dieser Hausübung gingen ebenfalls als Bonuspunkte in die Bewertung ein.

\subsection{Konzeptbeschreibung 2017}

Die Veränderung zum Übungsmodus aus 2016 bestand darin, dass die Beispiele für die wöchentliche Übung als ausformuliertes pdf-Dokument anstatt als online Beispiele zur Verfügung gestellt wurden. Dies hatte natürlich zur Folge, dass Studierende in der Übungsvorbereitung lediglich mit den statischen Beispielen und dem
Skriptum beschäftigt waren. In der Klausur als auch der Prüfung wurden aber variierende online Beispiele gegeben. Diese Änderung wird in der Evaluation im nächsten Kapitel näher diskutiert.

\subsection{Konzeptbeschreibung 2018}

Im Jahr 2018 wurden diese Übungsbeispiele dann wieder als online Assignment abgebildet. Zusätzlich wurden weitere, zum Vorlesungsstoff passende Beispiele thematisch zur Verfügung gestellt.

In allen drei Jahrgängen, wurde die Vorlesungsprüfung aus dem Pool der Übungsbeispiele mit teilweise geringfügigen Adaptionen zusammengestellt. Im folgenden Kapitel wird der Erfolg dieser Prüfung unter diversen Nebenbedingungen genauer beleuchtet.

\section{Evaluation}

In den ersten zwei Diagrammen werden Aktivitäten und Ergebnisse der Übungen aus dem Sommersemester 2018 und 2019 dargestellt. Die darauffolgenden Histogramme stellen die Übungs- und Prüfungserfolge der Jahrgänge 2016 bzw. 2017 dar. Die Tabellen am Ende des Kapitels führen den Zusammenhang der einzelnen Übungselemente mit der positiven bzw. negativen Vorlesungsprüfung näher aus.

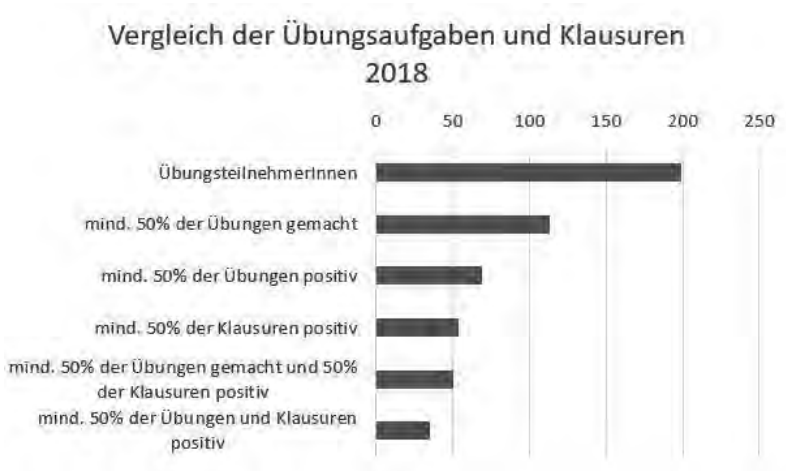

Abbildung 1: Vergleich der Übungsaufgaben und der Klausuren der Übung 2018S 


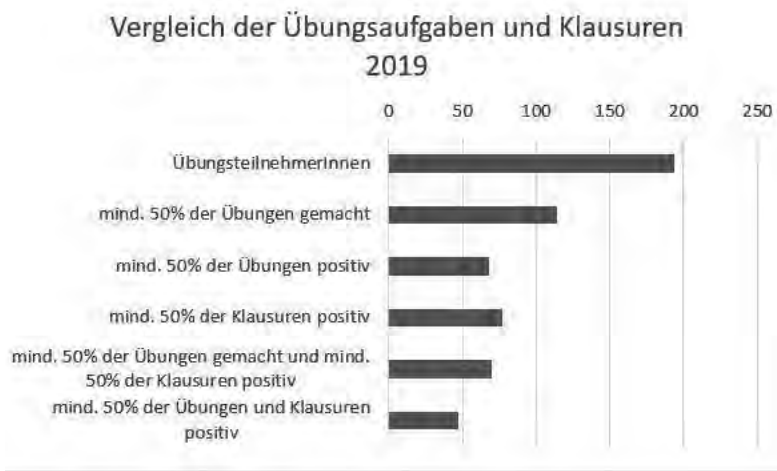

Abbildung 2: Vergleich der Übungsaufgaben und der Klausuren der Übung 2019S

Bei der Gegenüberstellung der Übungsaufgaben und der Klausuren fällt auf, dass in beiden Jahrgängen 2017 und 2018 die Zahl jener Studierenden, die mindestens 2 Klausuren erfolgreich abschließen konnten, nicht sehr von der Zahl jener, die zusätzlich die Hälfte der Übungsaufgaben gemacht haben, abweicht. Im Abbildung 2 stimmt letztere sogar mit der Anzahl der Studierenden, die mindestens die Hälfte der Übungsaufgaben positiv absolviert haben, beinahe überein. Dieser Vergleich lässt den Schluss $\mathrm{zu}$, dass eine positive Korrelation zwischen Übungsaufgaben und erfolgreich abgelegten Klausuren besteht.

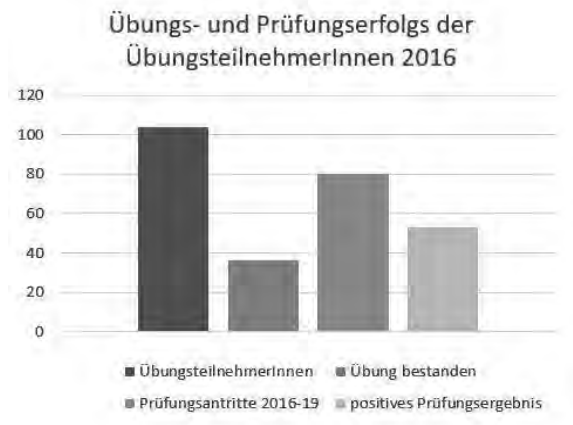

Abbildung 3: Vergleich des Übungs- und Prüfungserfolgs der ÜbungsteilnehmerInnen 2016

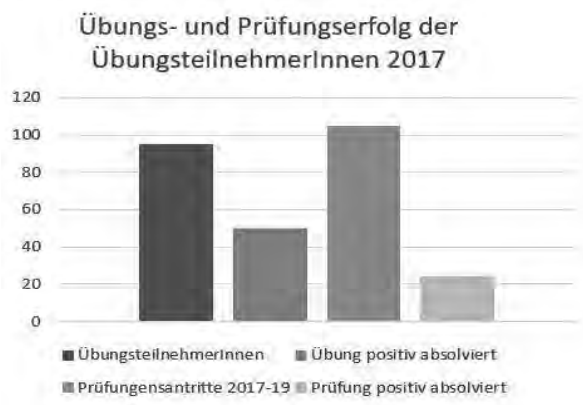

Abbildung 4: Vergleich des Übungs- und Prüfungserfolgs der ÜbungsteilnehmerInnen 2017
Der Vergleich der Jahre 2016 und 2017 zeigt, dass eine höhere Abschlussquote der Übung nicht auf eine höhere Erfolgsrate der Prüfungen schließen lässt. So haben in der Übung des Sommersemesters 2016, siehe Abbildung 3, nur 36 von 104 TeilnehmerInnen die Übung erfolgreich beendet, jedoch ist der Prüfungserfolg bei 53 positiven Ergebnissen von 80 deutlich höher. Gegenteiliges ist beim Vergleich des Übungs- und Prüfungserfolgs der Studierenden im Sommersemester 2017 zu erkennen, siehe Abbildung 4. Der Anteil der positiven Prüfungsergebnisse liegt bei fast $24 \%$, während der Anteil jener ÜbungsteilnehmerInnen, die die Übung bestanden haben, bei fast $53 \%$ liegt.

\begin{tabular}{llll}
\hline Übung 2016S & $\begin{array}{l}\text { Prüfung } \\
\text { positiv }\end{array}$ & $\begin{array}{l}\text { Prüfung } \\
\text { negativ }\end{array}$ & gesamt \\
\hline $\begin{array}{l}\text { Prüfungsantritte gesamt } \\
\begin{array}{l}2016-19 \text { inklusive Mehrfa- } \\
\text { chantritte }\end{array}\end{array}$ & $38.76 \%$ & $61.24 \%$ & 485
\end{tabular}

Prüfungsantritte von Teilneh-

merlnnen der Übung inklusive

\begin{tabular}{|c|c|c|c|}
\hline Mehrfachantritte & $61.25 \%$ & $38.75 \%$ & 80 \\
\hline TeilnehmerInnen der Übung * & $65.38 \%$ & $34.62 \%$ & 104 \\
\hline TeilnehmerInnen der Übung & $50.96 \%$ & $49.04 \%$ & 104 \\
\hline $\begin{array}{l}70 \% \text { der Übungsaufgaben } \\
\text { angesehen }\end{array}$ & $63.41 \%$ & $36.59 \%$ & 41 \\
\hline 2 Klausuren bestanden & $69.45 \%$ & $30.55 \%$ & 36 \\
\hline 3 Klausuren bestanden & $100 \%$ & $0 \%$ & 4 \\
\hline
\end{tabular}

$\begin{array}{cccc}3 \text { Klausuren bestanden } & 100 \% & 0 \% & 4 \\ \text { *Die Ergebnisse beziehen sich auf mindestens einmal angetreten }\end{array}$ (erste Spalte) bzw. nie angetreten (zweite Spalte).

Tabelle 1: Vergleich des Prüfungserfolgs der TeilnehmerInnen der Übung 2016S mit Übungskomponenten.

\begin{tabular}{|c|c|c|c|}
\hline Übung 2017S & $\begin{array}{l}\text { Prüfung } \\
\text { positiv }\end{array}$ & $\begin{array}{l}\text { Prüfung } \\
\text { negativ }\end{array}$ & gesamt \\
\hline $\begin{array}{l}\text { Prüfungsantritte gesamt } \\
\text { 2017-19 inklusive Mehrfa- } \\
\text { chantritte }\end{array}$ & $32.42 \%$ & $67.58 \%$ & 309 \\
\hline $\begin{array}{l}\text { Prüfungsantritte von Teilneh- } \\
\text { merlnnen der Übung inklusive } \\
\text { Mehrfachantritte }\end{array}$ & $22.86 \%$ & $77.14 \%$ & 105 \\
\hline TeilnehmerInnen der Übung * & $77.89 \%$ & $22.11 \%$ & 95 \\
\hline TeilnehmerInnen der Übung & $25.26 \%$ & $74.74 \%$ & 95 \\
\hline 2 Klausuren bestanden & $21.05 \%$ & $78.95 \%$ & 19 \\
\hline 3 Klausuren bestanden & $29.03 \%$ & $70.97 \%$ & 31 \\
\hline
\end{tabular}

Tabelle 2: Vergleich des Prüfungserfolgs der TeilnehmerInnen der Übung $2017 S$ mit Übungskomponenten. 


\begin{tabular}{llll}
\hline $\begin{array}{l}\text { Übung 2018S } \\
\text { Prüfungsantritte gesamt }\end{array}$ & $\begin{array}{l}\text { Prüfung } \\
\text { positiv }\end{array}$ & $\begin{array}{l}\text { Prüfung } \\
\text { negativ }\end{array}$ & gesamt \\
\hline $\begin{array}{l}\text { 2016-19 inklusive Mehrfa- } \\
\text { chantritte }\end{array}$ & $39.92 \%$ & $60.08 \%$ & 238 \\
$\begin{array}{l}\text { Prüfungsantritte von Teilneh- } \\
\text { merlnnen der Übung inklusive }\end{array}$ & & & \\
$\begin{array}{l}\text { Mehrfachantritte } \\
\text { Teilnehmerlnnen der Übung * }\end{array}$ & $42.41 \%$ & $57.59 \%$ & 158 \\
$\begin{array}{l}\text { Teilnehmerlnnen der Übung } \\
\text { 70\% der Übungsaufgaben }\end{array}$ & $33.67 \%$ & $66.33 \%$ & 199 \\
$\begin{array}{l}\text { angesehen } \\
\text { 2 Klausuren bestanden }\end{array}$ & $48.45 \%$ & $51.55 \%$ & 97 \\
\hline Klausuren bestanden & $63.43 \%$ & $68.57 \%$ & 35 \\
\hline
\end{tabular}

* Die Ergebnisse beziehen sich auf mindestens einmal angetreten (erste Spalte) bzw. nie angetreten (zweite Spalte).

Tabelle 3: Vergleich des Prüfungserfolgs der Teilnehmerlnnen der Übung 2018 S mit Übungskomponenten.

In den Tabellen 1, 2 und 3 wurden die Auswirkungen der einzelnen Übungselemente auf das Prüfungsergebnis untersucht. Während von insgesamt 485 Antritten in den Jahren 2016 bis 2019 fast 39\% positiv und 61.24\% negativ waren, fielen $61.25 \%$ der 80 Antritte von ÜbungsteilnehmerInnen im selben Zeitraum positiv aus, siehe Tabelle 1. Die Ergebnisse der gesamten Kohorte zeigen ein gegenteiliges Verhalten im Vergleich zu den Übungsteilnehmern. Ebenfalls kann aus Tabelle 1 abgelesen werden, dass viele jener Studierenden, die gute Ergebnisse bei den Übungsaufgaben und den Klausuren erreichen konnten, auch die Prüfung positiv abschließen konnten. Je mehr Übungselemente von den Studierenden behandelt wurden, desto höher ist der Prozentsatz der erfolgreichen Prüfungen. So haben zum Beispiel in der Übung im Jahr 2016 von 41 Studierenden, die 70\% der Übungsaufgaben gemacht haben, 63.41\% die Prüfung bestanden. Von jenen, die auf 2 Klausuren mindestens die Hälfte der Punkte erreicht haben, waren es $69.45 \%$ und von jenen, die 3 Klausuren bestanden haben, 100\%.

Die Übung des Sommersemesters 2017 zeigt ähnliche Ergebnisse, siehe Tabelle 2. Der Prozentsatz der Studierenden, die an der Übung teilgenommen und die Prüfung bestanden haben, ist zwar geringer als im Vorjahr, jedoch steigt der Anteil der Studierenden mit positivem Prüfungsergebnis mit der Anzahl der bestandenen Klausuren.

Dieses Verhalten ist auch in Tabelle 3 zu erkennen. Der Prozentsatz der Studierenden, die in mehr als drei Klausuren und in der Prüfung 50\% der Punkte errreichen konnten, ist mehr als doppelt so hoch wie jener, der sich auf 2 Klausuren bezieht.
Im Jahr 2016 konnte die Hälfte der ÜbungsteilnehmerInnen die Prüfung positiv abschließen, während es 2018 zirka ein Drittel war. Zu erwähnen ist allerdings, dass bei vielen ÜbungsteilnehmerInnen erst in den Folgejahren die Vorlesungsprüfung abgelegt wird. Da für diesen Beitrag nur Daten aus den Jahren 2016 bis 2019 miteinbezogen wurden, liegen für den Jahrgang 2018 noch vergleichsweise wenige Prüfungsdaten vor.

\section{Conclusio}

Aus den erhobenen Daten lässt sich eine positive Korrelation zwischen einem erfolgreichen Abschluss der Übung und dem Bestehen der Vorlesungsprüfung feststellen. Studierende, die die Lehrveranstaltung bei einem Vortragenden mit der zur Vorlesung angepassten Übung besucht haben, schneiden im Vergleich besser ab, als jene, die lediglich das Angebot der Vorlesung wahrgenommen haben. Die nicht so eindeutigen Ergebnisse des Jahrgangs 2017 könnten darauf zurückgeführt werden, dass dies der einzige Jahrgang ist, welcher statische Übungsaufgaben zur Verfügung gestellt bekommen hat. Dies könnte in der Folge dazu geführt haben, dass Studierende den statischen Beispielen zu viel Relevanz in der Prüfungsvorbereitung zugesprochen und dadurch die variierenden online Beispiele vernachlässigt haben.

Im Großen und Ganzen bestätigen die Ergebnisse allerdings die These, dass Lehrveranstaltungen als abgestimmtes System aus Vorlesung und Übung geplant werden sollten. Die Abstimmung der unterschiedlichen Elemente ist hierbei essentiell. Die Daten haben bestätigt, dass, im Sinne des „Constructive Allignments“, sowohl Übungen, als auch Klausuren auf die Prüfung einerseits inhaltlich andererseits auch methodisch vorbereiten sollen.

\section{References}

[1] O. Uner und H. L. Roediger, „,The Effect of Question Placement on Learning from Textbook Chapters“, Journal of Applied Research in Memory and Cognition, Bd. 7, Nr. 1, S. 116-122, März 2018, doi: 10.1016/j.jarmac.2017.09.002.

[2] H. L. Roediger und A. C. Butler, „The critical role of retrieval practice in long-term retention“, Trends in Cognitive Sciences, Bd. 15, Nr. 1, S. 20-27, Jan. 2011, doi: 10.1016/j.tics.2010.09.003.

[3] A. Körner, S. Winkler, R. Leskovar, F. Gorgas: "OnlineKomponenten der Lehre an der TU Wien", in: Tagungsband ASIM 2018, 24. Symposium Simulationstechnik, ISBN: 978-3-901608-12-4; 65 S. 